\title{
Rapid Detection of Bacterial Endotoxins in Ophthalmic Viscosurgical Device Materials by Direct Analysis in Real Time Mass Spectrometry
}

\begin{abstract}
Hongli $\mathrm{Li}^{1,2}$, Victoria M. Hitchins ${ }^{1}$, Samanthi Wickramasekara ${ }^{* 1}$
1. Division of Biology, Chemistry, and Materials Science, Center for Devices and Radiological Health, US Food and Drug Administration, Silver Spring, Maryland, 20993, United States.

2. Jiangsu Collaborative Innovation Center of Biomedical Functional Materials, College of Chemistry and Materials Science, Nanjing Normal University, Nanjing 210023, China
\end{abstract}

*Corresponding Author: Samanthi Wickramasekara, Ph.D, Division of Biology, Chemistry and Materials Science (DBCMS), Center for Devices and Radiological Health (CDRH), Food and Drug Administration. 10903 New Hampshire Avenue, Bldg 64, Rm 3064, Silver Spring, MD 20993. Email: Samanthi.Wickramasekara@fda.hhs.gov Phone: 301-796-2475. Fax 201-7969826

(C) 2016. This manuscript version is made available under the Elsevier user license http://www.elsevier.com/open-access/userlicense/1.0/ 


\begin{abstract}
Bacterial endotoxins are lipopolysaccharides bound to the bacterial cell wall and released when bacteria rupture or disintegrate. Possible contamination of endotoxin in ophthalmic devices can cause a painful eye inflammation or result in toxic anterior segment syndrome after cataract surgery. Measurement of bacterial endotoxin in medical device materials is difficult since endotoxin binds with polymer matrix and some of the materials are very viscous and non-water soluble, where traditional enzyme-based Limulus amoebocyte lysate (LAL) assay cannot be applied. Here we propose a rapid and high throughput ambient ionization mass spectrometric (MS) method using direct analysis in real time (DART) for the evaluation of endotoxin contamination in medical device materials. Large and structurally complex endotoxin instantaneously breaks down into low-mass characteristic fragment ions using DART and is detected by MS in both positive and negative ion modes. This method enables the identification and separation of endotoxin from medical materials with a detection limit of $0.03 \mathrm{ng} \mathrm{mL}^{-1}$ endotoxins in aqueous solution. Ophthalmic viscosurgical device materials including sodium hyaluronate (NaHA), non-water soluble perfluoro-n-octane (PFO) and silicone oil (SO) were spiked with different known concentrations of endotoxin and analyzed by DART MS, where the

presence of endotoxin was successfully detected and featured small mass fragment ions were generated for NaHA, PFO and SO as well. Current findings showed the feasibility of measuring endotoxin contamination in medical device materials using DART-MS, which can lead to a onestep analysis of endotoxins in different matrices, avoiding any potential contamination during sample pre-treatment steps.
\end{abstract}

Key words: endotoxin, sodium hyaluronate, perfluoro-n-octane, silicone oil, direct analysis in real time, mass spectrometry. 


\section{Introduction}

Lipopolysaccharides (LPS), also known as bacterial endotoxins, are located in the outer membrane of gram-negative bacteria, and are structural lipoglycans with molecular weight of 10$40 \mathrm{kDa}$. Endotoxins are composed of three main parts: (1) O-antigen section of repeating sugar units; (2) core oligosaccharide region containing heptose and 3-deoxy-D-mannooctulosonic acid (known as KDO) sugars as well as non-carbohydrate components; and (3) lipid A portion with multiple fatty acid chains connected through phosphorylated $\mathrm{N}$-acetyl glucosamine disaccharide, where the hydrophobic lipid A domain anchors into the bacteria membrane. [1-4] Endotoxin or LPS serves as a protection barrier for bacteria from its surroundings. However, they produce multiple negative biological effects when released into the mammalian's bloodstream, such as development of inflammatory response, invasion of the immune system, sepsis, septic shock, adult respiratory distress syndrome, and disseminated intravascular coagulation [5-7]. It is also known as a pyrogen or heat-stable toxin, due to its fever causing effect [8].

As of today, endotoxin contamination has been reported and studied in a wide range of circumstances,[9-12] and it remains a serious threat to the public health. For example, the outbreak of toxic anterior segment syndrome (TASS) in 2005 involved 112 patients,[13] caused by use of an endotoxin-contaminated device during cataract surgery, has greatly increased people's attention and awareness of the risks of endotoxin contamination in medical devices. TASS induces toxic damage to intraocular tissues, normally resulting in inflammation, blurry vision, ocular pain, eye redness or even permanent corneal endothelial and irregular dilated pupil within 24 hours of cataract surgery.[14-17] The main cause for TASS can be due to the endotoxin contamination of ophthalmic surgical instruments, supplies, accessories, intraocular solutions etc., during manufacturing, sterilization, or packaging processes [13, 18-21]. Retinal tamponades are ophthalmic viscosurgical devices (OVD), which are viscous and viscoelastic intraocular fluids used during a variety of surgeries to create and maintain space in the anterior chamber to protect internal eye tissues. However, they may be contaminated with endotoxin as well $[19,20]$. Due to the serious risks associated with endotoxin, the U.S. Food and Drug Administration (FDA) has issued endotoxin testing recommendations for intraocular single-use ophthalmic devices for industry. According to the FDA guidance, [22] the recommended 
endotoxin limit for release testing is $\leq 0.2 \mathrm{EU}$ (endotoxin units) $\mathrm{mL}^{-1}$, regardless of site of use (anterior or posterior segment).

Measuring endotoxin in different matrix and medical device materials is critical for assessing a product's safety. The endotoxin limit for devices that are in contact with blood is 20 EU/device and for devices in contact with cerebrospinal fluid it is $2.15 \mathrm{EU} \mathrm{mL}^{-1}$. Currently, the most widely used method for endotoxin or LPS detection and quantification is the Limulus Amebocyte Lysate (LAL) assay that is based on the enzymatic activity between amoebocytes extracted from horseshoe crab and bacterial endotoxin [23-25]. However, this methodology measures endotoxin indirectly; the procedure involves several steps, reaction takes place in aqueous environment, and there are other interfering compounds reacting with LAL reagent[26]. Thus, this test cannot be applied to detect the presence of endotoxin in water-insoluble materials.

Alternatively, a wide variety of mass spectrometry (MS)-based techniques has been applied for the detection of complex LPS or lipid A [27-30]. For example, Caroff et al. reported direct micro-extraction and analysis of intact LPS by combined thin-layer chromatography and matrix-assisted laser desorption ionization (MALDI) MS [27]. ESI-MS ${ }^{\mathrm{n}}$ have been used for the analysis of lipid A isolated from LPS as well [28]. These studies were mainly focused on the structural characterization of intact or partial LPS, but the convenience of traditional ion sources (such as ESI, MALDI) are limited by their speed, sample status (gas, liquid and solid) or sample introduction conditions (in matrix or/and under vacuum). Instead, other approaches for the measurement of LPS using MS are to fractionate intact LPS, extract specific fatty acids and derivatize them prior to analysis. Gas chromatography (GC)- or liquid chromatography (LC) coupled to MS is then applied for the separation, detection and quantitation of targeted fatty acid [30-32]. These methods can also be applied to carbohydrate-containing samples, which normally interfere with endotoxin biological assays but still suffer from multiple sample pre-treatment steps and tedious, time-consuming method development. The LOD lies in the range of a few to tens of $\mathrm{ng} \mathrm{mL^{-1 }}$, or even $\mu \mathrm{g} \mathrm{mL}^{-1}$. Technique and methodology improvements for rapid, simple and high throughput determination of bacterial endotoxin in medical devices of different materials are preferred for product safety assessment as well as emergency situations. Replacing traditional ionization sources and incorporating novel sample introduction and ionization technique on MS may be a faster and more accurate method to measure endotoxins. 
As an ambient ionization source, direct analysis in real time (DART) can ionize gases, liquids and solids in open air instantaneously, where ionization can happen on sample surface directly, requiring none or little sample preparation $[33,34]$. When the DART source is coupled to different mass spectrometers, it provides a rapid MS analysis of a wide range of compounds [35-39], where its mechanism and applications are described elsewhere and summarized in a couple of review papers [33,34]. In this study DART-MS is introduced for the first time to measure the presence of bacterial endotoxin in OVD materials, including sodium hyaluronate (NaHA),[40] perfluoro-n-octane (PFO) [41, 42], and silicone oil (SO) [43, 44]. Sodium hyaluronate is currently the most popular viscoelastic injection for a variety of intraocular surgical procedures such as cataract extraction, lens implantation and retina attachments since the 1970s. In contrast, PFO is a low-viscosity dispersive retinal tamponade and SO is a highdensity cohesive retinal tamponade. These two materials are selected for this study because they are both water-insoluble materials, and the detection of endotoxin contamination in them using traditional LAL assay or other aqueous-based tests remains a challenge. Using OVD materials as examples, the results obtained in this study suggest a rapid, simple, direct, sensitive and high throughput analytical methodology for endotoxin measurement. It is based on the detection of structural identities of endotoxin directly; adopts one-step experimental protocol not requiring additional chemicals or reagent kits; and the analysis time is within seconds with minimum or no sample preparation, providing an endotoxin LOD of $0.03 \mathrm{ng} \mathrm{mL}^{-1}$ in aqueous solution, which is approximately $0.3 \mathrm{EU} \mathrm{mL}^{-1}$.

\section{Experimental}

\subsection{Chemicals and sample preparation}

Control standard endotoxin (CSE, Lot \# 249024) was purchased from Associates of CAPE COD, Inc. (East Falmouth, MA). Each vial contained 10 ng lyophilized and long freezedried endotoxin derived from E. Coli O113: H10. Limulus amebocyte lysate (LAL) reagent water manufactured by Associates of CAPE COD Inc., (East Falmouth, MA) was used to reconstitute CSE. It contained less than $0.0001 \mathrm{EU} \mathrm{mL}^{-1}$ endotoxin and $1.56 \mathrm{pg} \mathrm{mL} \mathrm{m}^{-1}(1,3)-\beta$-D-glucan. Methanol (Chromasolv, HPLC grade $\geq 99.9 \%$ ) was purchased from Sigma-Aldrich (St. Louis, MO) and Hexane (Optima ${ }^{\mathrm{TM}}$ ) was obtained from Fisher Scientific (Pittsburgh, PA). Pyroclear precision pipette tips supplied from Associates of CAPE COD Inc., (certified free of interfering 
endotoxins and glucans) were used to transfer and prepare samples in this work. $2 \mathrm{~mL}$ LC-MS injection vials from Waters, Inc., (Milford, MA) were used as sample containers and were heated at $250^{\circ} \mathrm{C}$ for 30 mins for depyrogenation before use. LAL reagent water $0.5 \mathrm{~mL}$ was added to a vial of dry CSE to prepare $20 \mathrm{ng} \mathrm{mL}^{-1}$ endotoxin standard stock solution. A series of dilutions was then performed with 50: 50 methanol: water to prepare different CSE solutions of 0.031, $0.063,0.16,0.31,0.47,0.63,1.25,3.1$ and $10 \mathrm{ng} \mathrm{mL}^{-1}$. CSE solutions were stored at $4-8^{\circ} \mathrm{C}$ for analysis in 4 days, and fresh CSE solutions were prepared afterwards. Ophthalmic viscosurgical device (OVD) intraocular fluids analyzed in this study include Healon, Silikon and Perfluoron, which are all prescription medical products. Healon manufactured by advanced medical optic Uppsala $\mathrm{AB}$, Sweden contains $0.4 \mathrm{~mL}$ of $10 \mathrm{mg} \mathrm{mL}^{-1}$ sodium hyaluronate (NaHA). Perfluoron, purified perfluoro-n-octane (PFO) liquid, was purchased from Alcon Laboratories, Inc., (Fort Worth, TX) and was used directly without further processing. Silikon 1000 from Alcon Laboratories, Inc. is packed with $8.5 \mathrm{~mL}$ purified silicone oil. Healon and Silikon products are highly viscous fluids. Single-use syringes were used to transfer the fluids for further dilutions. Healon solutions were prepared with a 1: $5(\mathrm{w}: \mathrm{w})$ ratio in 50:50 (v:v) water: methanol. Silikon was diluted in hexane with a 1:100 (w:w) ratio. Equal volumes of CSE solutions and 1: $5(\mathrm{w}: \mathrm{w})$ dilution of NaHA solution were mixed together to prepare endotoxin spiked Healon sample, resulting in final concentrations of $0.31,0.47,0.63,1.55,5$ and $10 \mathrm{ng} \mathrm{mL}{ }^{-1}$ endotoxin in the mixture. Due to the solvent immiscibility of CSE solution, PFO and hexane, endotoxin-spiked PFO and SO samples were prepared in different ways. In detail, $1 \mu \mathrm{L}$ of CSE solutions of different concentrations including $0.063,0.16,0.31,0.63$ and $1.25 \mathrm{ng} \mathrm{mL}^{-1}$ and $1 \mu \mathrm{L}$ of PFO fluid or Silikon solutions were pipetted on top of each other to the sample tip to make the endotoxin containing OVD samples.

\subsection{Sample analysis by direct analysis in real time mass spectrometry}

All CSE and OVD standards including sodium hyaluronate (NaHA), PFO and silicone oil, and samples of endotoxin containing OVD fluids were analyzed by direct analysis in real time mass spectrometry. Direct analysis in real time (DART, SVP-201) controller from IonSense, Inc., (Saugus, MA) was connected to the LTQ XL linear ion trap (Thermo Scientific, Waltham, MA) mass spectrometer (MS) through Vapur ${ }^{\mathrm{TM}}$ interface (IonSense, Inc.). The DART controller was operated with $99.999 \%$ purity helium gas (Roberts Oxygen, Rockville, MD) at a temperature of 
$400{ }^{\circ} \mathrm{C}$ in both positive and negative ion modes. Standard samples were introduced by a 12 DIPit holder (IonSense, Inc.) on a moving rail, which allowed multiple liquid samples to be analyzed automatically in a sequence using a web-based DART software (version 5.0.5). Within the programmed sequence run, the heat waiting time was set to 30 seconds, contact closure delay time was set to 1 second and the rail moving speed was set at $0.3 \mathrm{~mm} / \mathrm{s}$. Dip-it tips were heated at $400{ }^{\circ} \mathrm{C}$ for $\sim 10$ seconds prior to sample loading for in-depth cleaning in order to avoid any potential contamination from the glass capillary tip. For accurate quantification, $2 \mu \mathrm{L}$ (unless specified) of CSE samples, diluted NaHA standards as well as endotoxin-spiked NaHA solutions were pipetted on to the bottom of the dip-it tip (glass capillary tube) and analyzed in both positive and negative ionization modes by DART-MS using a moving rail. In contrast, when PFO samples were introduced into the DART MS using the linear rail with the holder, the sample evaporated completely by the time it moved to the center position of the source due to its low boiling point $\left(103-104^{\circ} \mathrm{C}\right)$. Alternatively, undiluted $2 \mu \mathrm{L}$ of PFO standard and endotoxincontaining PFO samples ( $1 \mu \mathrm{L}$ each pipetted on top of each other) were placed at the source center manually and analyzed immediately after sample loading in negative ionization mode. Diluted SO standards in hexane were introduced and analyzed in the same way as CSE samples in positive mode, while the endotoxin and SO mixture $(1 \mu \mathrm{L}$ of CSE samples in 50:50 water: methanol and $1 \mu \mathrm{L}$ of 1 to 100 dilution of silicone oil in hexane spotted separately on top of each other) were air dried on the holder prior to the analysis by DART-MS in positive mode. Themo tune plus software (version 2.6.0, Thermo Scientific) was used to acquire mass spectrometer data. The capillary temperature of MS was at $200^{\circ} \mathrm{C}$ in both ionization modes. The mass range of 1001200 was employed for all samples except SO, where extended mass range up to 2000 was used. Capillary voltage and tube lens were $25 \mathrm{~V}, 100 \mathrm{~V}$ and $-50 \mathrm{~V},-120 \mathrm{~V}$ for positive and negative ion mode, respectively. To enhance the sensitivity of small-mass fragments for endotoxin-spiked NaHA samples, MS parameters were tuned to the following numbers: capillary temperature was $300^{\circ} \mathrm{C}$ in both modes, capillary voltage and tube lens were $15 \mathrm{~V}, 100 \mathrm{~V}$ in positive ion mode and $-24,-100 \mathrm{~V}$ in negative mode. Additionally, source fragmentation of $30 \mathrm{~V}$ was employed for both PFO standard and endotoxin-mixed PFO samples for structural characterization of PFO. Sheath/sweep/auxiliary gas flow rates were set at 0 for all analysis in this study. Multiple replicate $(\geqslant 3)$ analyses were performed for every single sample. It is worthy to mention that the evaluation of endotoxin and endotoxin contamination in NaHA using DART-MS were 
conducted in both positive and negative modes. Analyses of PFO and its endotoxin spiked sample were performed in negative ionization mode only, since no reasonable signal was generated for PFO in positive mode. Transposed phenomenon was observed for silicone oil which gives stable signal only in positive ionization mode.

In order to confirm the identified/assigned ions for the endotoxin standard, experiments were repeated with DART SVP attached to Agilent 6540B accurate mass Q TOF mass spectrometer (Agilent Technologies, Santa Clara, CA, USA) in both positive and negative ionization modes. (Additional experimental parameters can be found in Supplemental Information).

\subsection{Data analysis}

Xcalibur 2.1 software (Thermo Scientific) was used to analyze the data collected from mass spectrometer. Ion intensities were obtained after spectral averaging across the total ion chromatogram during the sample exposure to DART beam followed by background subtraction. The mass spectra collected from DART dip-it tips only with corresponding solvents were considered as the background. All final MS figures as well as calibration curves were generated in Origin 9 (Northampton, MA) using the mass spectral peak lists exported from Xcalibur 2.1, where the y scales of displayed MS spectra were normalized to $100 \%$ using the base peak, but the absolute mass intensities of specific $\mathrm{m} / \mathrm{z}$ from different endotoxin concentrations were used to plot calibration curves. All chemical structures were drawn using ChemBioDraw Ultra 13.0 (cambridgesoft.com, MA, USA) and the structures were manually assigned based on the $\mathrm{m} / z$ values obtained.

\section{Results and Discussion}

\subsection{Endotoxin standard analysis and quantification}

As shown in Fig. 1A, bacterial endotoxin adopts a complex chemical structure, starting from O-antigen, connected through core-oligosaccharide and ending with lipid $\mathrm{A}$ in the cell interior, where lipid $\mathrm{A}$ is believed to be responsible for a majority of the biological activities[7, 28]. Endotoxin or LPS has the polysaccharide chain with various units of hexose, $N$-acetylhexosamine, heptose, deoxyoctonic acid, phosphate and amine structure as well as 4-6 fatty acid chains with 12-14 carbons. The large molecular weight of the intact endotoxin makes it difficult to be analyzed using traditional ionization techniques coupled to mass spectrometry such as ESIMS. These analyses require high-mass range instruments and produce high $\mathrm{m} / \mathrm{z}$ ions with low 
$\mathrm{S} / \mathrm{N}$ ratio, resulting in mass spectral peaks that are difficult to be interpreted for chemical structure information. For analysis of aqueous samples using DART, CSE standards are simply transferred by pipetting $2 \mu \mathrm{L}$ solutions onto the capillary tip for quantitative purpose or the capillary tip can be dipped into the endotoxin solution directly for qualitative analysis. DART ionization with different helium temperatures of $250^{\circ} \mathrm{C}, 300^{\circ} \mathrm{C}, 350^{\circ} \mathrm{C}, 400^{\circ} \mathrm{C}$ and $450^{\circ} \mathrm{C}$ for endotoxin in both the positive and negative ionization modes was evaluated and the results are shown in Fig. S1. Starting at low temperature of $250^{\circ} \mathrm{C}$, small-mass endotoxin fragments were detected but with lower $\mathrm{S} / \mathrm{N}$ ratios. Signal intensity reached to a maximum at $400{ }^{\circ} \mathrm{C}$, and then slightly decreased at $450{ }^{\circ} \mathrm{C}$. Thus, a temperature of $400^{\circ} \mathrm{C}$ was employed for all the samples throughout this study. Figs. $1 \mathrm{~B}$ and $1 \mathrm{C}$ show the multiple characteristic $\mathrm{m} / \mathrm{z}$ ions of small fragments detected for endotoxin within seconds in both positive and negative ion modes, respectively. Table 1 lists the main fragments identified for the endotoxin standards in both positive and negative ionization mode using the LTQ-MS. Molecular formulas were assigned based on the accurate mass values confirmed by the high resolution Q TOF mass measurements for endotoxin standards, where the high resolution mass spectra for endotoxin standards were attached in Fig. S2 in SI. DART can generate radical, cluster or/and protonated/deprotonated ions [33, 45], however, ammonium adduct is common for carbohydrates via DART ionization according to literature studies [46-50]. For example, $\mathrm{m} / \mathrm{z}, 198$ is identified as ammonium adduct of monosaccharide in positive mode, $\left[\mathrm{M}+\mathrm{NH}_{4}\right]^{+}$; ions of $\mathrm{m} / \mathrm{z} 180$ were confirmed to be ammonium adduct of water loss product from hexose $\left[\mathrm{M}+\mathrm{NH}_{4}-\mathrm{H}_{2} \mathrm{O}\right]^{+} ; m / z$ values of 163,145 , 127 are protonated ions resulted from series of water loss from hexose as annotated (Table 1). Different peaks having lower counts in the range of $m / z$ 250-400 were also detected. They are disaccharide ammonium adduct or protonated water loss fragments from disaccharide (Table 1). . Correspondingly, monosacchride, disaccharide, and their water-loss fragments were detected for endotoxin as deprotonated ions in negative ion mode (Fig. 1C) as well. The proposed structures for ions of $m / z$ 117, 119 and 221 were included in Fig. S3 in supporting information. Altogether positive mode produced more $\mathrm{m} / \mathrm{z}$ ions compared to negative ion mode. The relative-intensity ratios among different ions vary $\pm 10 \%$ at specific experimental conditions, . Cation of $\mathrm{m} / \mathrm{z} 163$ and anion of $\mathrm{m} / \mathrm{z}, 179$ were then used to establish the calibration curves in positive (top right corner of Fig. 1B) and negative mode (top right corner of Fig. 1C), respectively, with varying endotoxin concentrations of $0.03,0.06,0.16,0.3,0.5,0.6$ and $1.25 \mathrm{ng} \mathrm{mL}^{-1}$. Polynomial 
calibration curves were fitted with higher $\mathrm{R}^{2}$ values over other type of curves; and the limit of detection (LOD) of $0.03 \mathrm{ng} \mathrm{mL} \mathrm{m}^{-1}$ with $\mathrm{S} / \mathrm{N}$ of 5 was obtained in both modes for bacterial endotoxin with $\geq 3$ replicates.

Based on the literature survey, Liu et.al. [47] and Smith et.al. [48] reported that monosaccharide and disaccharide standards also produce similar $\mathrm{m} / \mathrm{z}$ signals as observed for endotoxin in this study. The DART-MS fragmentation of endotoxin is similar to MS/MS spectra of glucose or MS spectra of disaccharides. Liu et. al also stated that high mass ion clusters were generally formed for the disaccharide and trisacchride at $450^{\circ} \mathrm{C}$ but DART-MS did not produce large molecular cluster ions $(>m / z 500)$ for the endotoxin standard with the current experimental setup. This mechanism of fragment ion formation for the endotoxin standard is similar to the glycosaminoglycan pyrolysis behavior at the DART interface as reported previously [51]. It is proposed that during the pyrolysis process the polysaccharide backbone of endotoxin breaks down into small sugar fragments by thermal decomposition. Many analytical assays have different interferences. For example, $\beta$-1, 3-glucan reacts with LAL reagent and needs to be avoided during assay protocol [26]. Though the saccharides can be interfering compounds for the detection of endotoxins by DART-MS, the technique involves none or little sample preparation and adopts nearly one-step protocol, which can limit the cross-contamination during analysis. In this study, large and complex endotoxin is decomposed into small fragments through DART within seconds, providing multiple mass identities associated with its structural features, which suggest a novel, simple, and high throughput method for the direct detection of endotoxins.

\subsection{Detection of endotoxin in sodium hyaluronate (NaHA)}

To validate the DART-MS method for potentially measuring endotoxin contamination in clinically used OVD materials, sodium hyaluronate (NaHA) was first selected as a test model. $\mathrm{NaHA}$ is the sodium salt of hyaluronic acid (HA), which is found naturally within the extracellular matrix within bodily tissues. Beside its application in the field of ophthalmology as dispensable viscoelastic substance in intraocular surgery, it is also used in cosmetics. Chemically $\mathrm{NaHA}$ is a polysaccharide containing repeating disaccharide unit of Na-glucuronate- $\mathrm{N}$ acetylglucosamine. Fig. 2A and 2B are mass spectra for the mixture of endotoxin and NaHA in positive and negative ion mode, respectively, for the sample containing $10 \mathrm{ng} \mathrm{mL}^{-1}$ endotoxin in NaHA solution. Similar to endotoxin, long chain of NaHA generated multiple small fragments in 
both ionization modes via DART-MS, as labeled with blue squares. Red squares indicate the low-mass ions identified for endotoxin standards as discussed above. Characteristic mass identities for endotoxin were successfully detected when mixed with NaHA. The disaccharide structural unit of NaHA (NaGlc-GlcNAc, shown on top of Fig. 2A) overlapped with a small structural component of endotoxin (Glc-GlcNAc, Fig. 1A), but the ions generated are clearly distinguishable. No identical fragments were observed based on the NaHA standard spectra (see Fig. S4 in SI). The major peaks observed for NaHA are associated with GlcNAc monomer such as [GlcNAc- $\mathrm{H}_{2} \mathrm{O}+\mathrm{H}^{+}$] at $m / z, 204$ and corresponding negative ion at $m / z$ 202, and a series of water-loss ions including positive ions of $\mathrm{m} / \mathrm{z} 168,186$ and negative ions of $\mathrm{m} / \mathrm{z} 166,184$. A fragment derived from GlcNAc was detected as well as annotated in Fig. 2. Zoomed windows show the resolution of endotoxin and NaHA mass ions with one mass unit difference, such as $\mathrm{m} / \mathrm{z} 126$ and 127 in positive mode and $\mathrm{m} / \mathrm{z} 142$ and 143 in negative mode. Notably, the LODs were achieved at $0.63 \mathrm{ng} \mathrm{mL}^{-1}$ and $0.47 \mathrm{ng} \mathrm{mL}^{-1}$ endotoxin in the mixture for positive and negative mode, respectively, which are higher than the LOD obtained for endotoxin standards at $0.03 \mathrm{ng} \mathrm{mL}^{-1}$ as shown above. This is probably due to the strong ion suppression effects of NaHA over endotoxin on DART. Overall, the successful detection of endotoxin in NaHA may lead to a new method for screening endotoxin contaminations in medical devices, and no chemical kits or tedious chemical or biological reactions are needed. The procedure is simple and the measurement is direct, avoiding potential endotoxin contamination in treatment steps, which are necessary using other methods.

\subsection{Detection of endotoxin in perfluoro-n-octane (PFO)}

Unique physical properties of PFO such as low viscosity, high vapor pressure, good optical clarity as well as immiscibility with water make PFO a favorable retinal tamponade fluid in vitreoretinal surgery. However, when 1-2 $\mu \mathrm{L}$ PFO standard was transferred onto a DART tip on a holder, the liquid totally evaporated before it arrived at the DART source via a moving rail. To overcome this problem, a capillary tube with PFO was manually placed to the center of DART source immediately after sample loading. PFO is fluorocarbon having molecular formula of $\mathrm{C}_{8} \mathrm{~F}_{18}$, and it forms a single $\left[\mathrm{C}_{8} \mathrm{~F}_{17} \mathrm{O}\right]^{-}$anion at $m / z, 435$ on DART as displayed in Fig. S5-A, which perhaps results from the reaction between PFO and $\mathrm{O}_{2}^{-}$produced by electron capture of atmospheric oxygen in negative DART as discussed elsewhere[45]. To further characterize PFO 
structure, a tandem MS spectrum, shown in Fig. S5-B, was obtained for PFO using 30V insource fragmentation energy, where a series of fragments with loss of $\mathrm{CF}_{2}$ were generated, providing more structural identities for PFO. It is worth noting that the fragment at $m / z, 119$ overlapped with one of the endotoxin mass identity at $\mathrm{m} / \mathrm{z}, 119$ listed in Table 1 , where high accurate mass spectrometer is needed to differentiate these two signals. Pipetting PFO liquid together with different concentrations of endotoxin solutions simulates the real endotoxin contaminated PFO sample. The results in Fig. S6 show that multiple endotoxin signals (annotated with red square) and PFO peaks are detected when endotoxin is incorporated into the device material. Fig. 3 displays the mass spectrum for the mixed sample of $1 \mu \mathrm{L}$ of $2.5 \mathrm{ng} \mathrm{mL}^{-1}$ endotoxin pipetted on top of $1 \mu \mathrm{L}$ of PFO with $30 \mathrm{~V}$ in-source collision energy applied. As displayed on the top panel of Fig. 3, two distinct DART total ion chromatogram (TIC) peaks with the total analysis time of $\sim 12$ seconds per sampling were observed for the mixed samples, which indicate PFO signal appeared slightly earlier the endotoxin signal. This is probably due to the low boiling point and high vapor pressure of the perfluoro-n-octane. PFO precursor ion with all its fragment ions was detected, and the structure interpretation was annotated with a major loss of $\mathrm{CF}_{2}$. While the intensity for endotoxin peaks decreased with collision energy, mass identities having low intensity beyond $\mathrm{m} / \mathrm{z} 200$ were not observed for endotoxin in this example. However, the major signals were clearly detected (labeled with red square), providing enough evidence for the presence of endotoxin. No additional small fragments were generated for endotoxin within the specified mass range. Moreover, no obvious signal suppression was observed for the mixed samples, and the LOD was determined at $0.06 \mathrm{ng} \mathrm{mL}^{-1}$ endotoxin with 1 $\mu \mathrm{L}$ sample when spiked with PFO. This is consistent to $2 \mu \mathrm{L} 0.03 \mathrm{ng} \mathrm{mL} \mathrm{m}^{-1}$ detection limit established for pure endotoxin standards in Fig. 1. Here the success of DART-MS technology in evaluating bacterial endotoxin contamination is demonstrated by detecting its fingerprinting signals in Perfloron device material, with or without collision energy, which could lead to an innovation to rapidly measure endotoxin substance in non-water soluble materials which cannot be achieved by traditional LAL assay.

\subsection{Detection of endotoxin in silicone oil}

Silikon 1000, in the form of silicone oil or polydimethylsiloxane, has been used as vitreous substitute to treat difficult cases of retinal detachment as well as medical devices for 
prosthetic and cosmetic purposes[43, 44]. Silicone injection is believed to be theoretically inert for decades but now is thought to be a potential cause for direct and indirect toxic effects in biological system[52-54]. The analysis of polydimethylsiloxane has been demonstrated by different techniques [55-57] with pyrolysis gas chromatography served as the preferred method, however, many of these techniques are involved with tedious method development and timeconsuming sample preparation steps. Gross J. H. et al.[58-60] showed the advantages of using DART-MS in analyzing silicone oil. Here, we are detecting endotoxin contamination in silicone oil, a high viscous, non-water soluble material directly by DART-MS. Mass spectrum of 1:100 weight dilution of SO in hexane is displayed in Fig. S7, in which a systematic pattern with mass difference of 74 was obtained up to $\mathrm{m} / \mathrm{z} 2000$. To mimic Silikon 1000 device contaminated with endotoxin, $1 \mu \mathrm{L}$ of endotoxin solution of different concentrations and $1 \mu \mathrm{L}$ 1:100 diluted silicone oil in hexane were pipetted on top of each other on the capillary tip of DART, and the sample was air-dried prior to analysis. Fig. 4 shows the mass spectrum of Silikon in the presence of $1 \mu \mathrm{L}$ of $1.25 \mathrm{ng} \mathrm{mL}^{-1}$ endotoxin in positive ion mode using DART. Two separate signal regions were formed with major identified endotoxin characteristic peaks detected below $\mathrm{m} / \mathrm{z}, 400$ (red square labelled) and a series of silicone oil pyrolysis fragments observed over $\mathrm{m} / \mathrm{z} 350$. Different fragments of $\mathrm{SO}$ are generated from the sequential loss of $\mathrm{SiO}\left(\mathrm{CH}_{3}\right)_{2}$, mass of 74, indicated by the unlabeled arrows in Fig. 4, where the intensity of Y-scale between $m / z$ 800-1150 is increased 15 -fold. For the mixture, the LOD was established at $1 \mu \mathrm{L} 0.06 \mathrm{ng} \mathrm{mL}^{-1}$ endotoxin in $\mathrm{SO}$, which is equivalent to $2 \mu \mathrm{L} 0.03 \mathrm{ng} \mathrm{mL}^{-1}$ endotoxin found for purified standards. In this analysis, $\mathrm{m} / \mathrm{z}$ ions of endotoxin and silicone oil were distributed into two different mass ranges, making DART-MS an ideal method for the detection of potential endotoxin contamination in silicone oil-based medical devices. Importantly, some OVD materials such as SO are water-insoluble, viscous, hard to handle and manipulate, and there are no chemical or biological tests readily available to measure endotoxin in such material or special sample treatment steps need to be developed. As demonstrated, DART is a direct-sampling and ionization technique. Medical device materials can be introduced to the source directly without any sample preparation and the test results can be readily obtained through a simple protocol.

\section{Conclusions}


In this work, DART-MS has been first applied for the analysis and assessment of endotoxin contamination in OVD materials. DART-MS analyses of endotoxin standards generated various characteristic mass identities in both positive and negative ion modes, fostering a LOD of $60 \mathrm{fg}$ endotoxin. When mixed with OVD models of NaHA, PFO and SO, endotoxin signature peaks were successfully detected in all spiked samples regardless of matrices , providing a new method to measure endotoxin in medical device materials having different physiochemical properties. On the other hand, DART simplified the analysis of large molecules or polymers using mass spectrometry, where pyrolysis or decomposition tends to happen as shown for the endotoxin, NaHA and SO. The methodology employing DART-MS adopts many advantages including rapid, simple, direct, sensitive, and especially, it involves no or minimum sample preparation. It is suitable for non-water soluble materials, which is a hindrance for most biological tests to direct measure bacterial endotoxin.

In the laboratory environment, different sources can introduce endotoxin contamination in medical products including glassware, chemical reagents, raw materials, buffers and the handling process, since endotoxin is prevalent in tap water, air and fingers. Thus endotoxin contamination is hard to avoid and cannot be ignored during the manufacturing process, which adds great complexity to endotoxin measurement in a wide range of circumstances. With the method developed here, it could potentially lead to a one-step high throughput and direct screening method for endotoxin contamination regardless of the sample status or types. There is no need to separate endotoxin from biomaterials, polymer or matrix, avoiding potential endotoxin contamination during treatment steps. It is also beneficial for volume- or quantitylimited samples and allows rapid analysis within seconds. All of these features make DART-MS an ideal candidate for measuring endotoxin contamination in other medical device materials or products.

\section{Acknowledgements and Disclaimer}

This work was supported by FDA Medical Countermeasure Initiative (MCMi) program and Division of Biology, Chemistry and Materials Science (DBCMS) fund. We thank Jason Brookbank for coordinating the grant for the DART source in this study as well as management support from Dr. Jose Centeno and Dr. Benita J. Dair. This project was supported in part by an appointment to the Research Participation Program at the Center for Devices and Radiological Health administrated by the Oak Ridge Institute for Science and Education through an interagency agreement between US Department of Energy and the US food and Drug Administration. 
The findings and conclusions in this paper have not been formally disseminated by the Food and Drug Administration and should not be constructed to represent any agency determination or policy. The mention of commercial products, their sources, or their use in connection with material reported herein is not to be construed as either actual or implied endorsement of such products by the Department of Health and Human Service.

\section{References}

[1] H. Dong, Q. Xiang, Y. Gu, Z. Wang, N.G. Paterson, P.J. Stansfeld, C. He, Y. Zhang, W. Wang, C. Dong, Structural basis for outer membrane lipopolysaccharide insertion, Nature, 511 (2014) 52-56.

[2] X. Wang, P.J. Quinn, Lipopolysaccharide: Biosynthetic pathway and structure modification, Prog Lipid Res, 49 (2010) 97-107.

[3] N. Kato, T. Sugiyama, S. Naito, Y. Arakawa, H. Ito, N. Kido, M. Ohta, K. Sasaki, Molecular structure of

bacterial endotoxin (Escherichia coli Re lipopolysaccharide): implications for formation of a novel heterogeneous lattice structure, Mol Microbiol, 36 (2000) 796-805.

[4] E.T. Rietschel, T. Kirikae, F.U. Schade, U. Mamat, G. Schmidt, H. Loppnow, A.J. Ulmer, U. Zahringer, U. Seydel, F. Dipadova, M. Schreier, H. Brade, Bacterial, Endotoxin - Molecular Relationships of Structure to Activity and Function, Faseb J, 8 (1994) 217-225.

[5] S. Cadenas, A.M. Cadenas, Fighting the stranger-antioxidant protection against endotoxin toxicity, Toxicology, 180 (2002) 45-63.

[6] M.R. Leonardo, R.A. Silva, S. Assed, P. Nelson-Filho, Importance of bacterial endotoxin (LPS) in endodontics, J Appl Oral Sci, 12 (2004) 93-98.

[7] M.B. Gorbet, M.V. Sefton, Endotoxin: The uninvited guest, Biomaterials, 26 (2005) 6811-6817.

[8] B. Beutler, E.T. Rietschel, Innate immune sensing and its roots: the story of endotoxin, Nat Rev Immunol, 3 (2003) 169-176.

[9] Z. Can, L. Wenjun, S. Wen, Z. Minglu, Q. Lingjia, L. Cuiping, T. Fang, Endotoxin contamination and control in surface water sources and a drinking water treatment plant in Beijing, China, Water Res, 47 (2013) 3591-3599.

[10] S.M. van Putten, M. Wubben, J.A. Plantinga, W.E. Hennink, M.J. van Luyn, M.C. Harmsen, Endotoxin contamination delays the foreign body reaction, J Biomed Mater Res A, 98 (2011) 527-534.

[11] M.K. Huntington, J.F. Williams, C.D. Mackenzie, Endotoxin contamination in the dental surgery, J Med Microbiol, 56 (2007) 1230-1234.

[12] Y. Nakagawa, T. Murai, C. Hasegawa, M. Hirata, T. Tsuchiya, T. Yagami, Y. Haishima, Endotoxin contamination in wound dressings made of natural biomaterials, J Biomed Mater Res B Appl Biomater, 66 (2003) 347-355.

[13] P.K. Kutty, T.S. Forster, C. Wood-Koob, N. Thayer, R.B. Nelson, S.J. Berke, L. Pontacolone, T.L. Beardsley, H.F. Edelhauser, M.J. Arduino, N. Mamalis, A. Srinivasan, Multistate outbreak of toxic anterior segment syndrome, 2005, J Cataract Refract Surg, 34 (2008) 585-590.

[14] J. Andonegui, L. Jimenez-Lasanta, D. Aliseda, F. Lameiro, [Outbreak of toxic anterior segment syndrome after vitreous surgery], Arch Soc Esp Oftalmol, 84 (2009) 403-405.

[15] M. Unal, I. Yucel, Y. Akar, A. Oner, M. Altin, Outbreak of toxic anterior segment syndrome associated with glutaraldehyde after cataract surgery, J Cataract Refract Surg, 32 (2006) 1696-1701.

[16] N. Mamalis, Toxic anterior segment syndrome, J Cataract Refract Surg, 32 (2006) 181-182.

[17] W.C. Hellinger, S.A. Hasan, L.P. Bacalis, D.M. Thornblom, S.C. Beckmann, C. Blackmore, T.S. Forster, J.F. Tirey, M.J. Ross, C.D. Nilson, N. Mamalis, J.E. Crook, R.E. Bendel, R. Shetty, M.W. Stewart, J.P. Bolling, 
H.F. Edelhauser, Outbreak of toxic anterior segment syndrome following cataract surgery associated with impurities in autoclave steam moisture, Infect Control Hosp Epidemiol, 27 (2006) 294-298.

[18] Z. Bodnar, S. Clouser, N. Mamalis, Toxic anterior segment syndrome: Update on the most common causes, J Cataract Refr Surg, 38 (2012) 1902-1910.

[19] S.Y. Buchen, D. Calogero, G. Hilmantel, M.B. Eydelman, Detecting endotoxin contamination of ophthalmic viscosurgical devices: intracameral versus intravitreal assays in rabbits, Ophthalmology, 119 (2012) e11-18.

[20] S.Y. Buchen, D. Calogero, G. Hilmantel, M.B. Eydelman, Rabbit Ocular Reactivity to Bacterial Endotoxin Contained in Aqueous Solution and Ophthalmic Viscosurgical Devices, Ophthalmology, 119 (2012) E4-E10.

[21] K.R. Kreisler, S.S. Martin, C.W. Young, C.W. Anderson, N. Mamalis, Postoperative inflammation following cataract extraction caused by bacterial contamination of the cleaning bath detergent, J Cataract Refract Surg, 18 (1992) 106-110.

[22] Endotoxin Testing Recommendations for Single-Use Intraocular Ophthalmic Device : Guidance for Industry and Food and Drug Administration Staff http://www.fda.gov/downloads/medicaldevices/deviceregulationandguidance/guidancedocuments/uc m393376.pdf, U. S. Food and Drug Administration 2015, pp. 1-9.

[23] P.A. Ketchum, T.J. Novitsky, Assay of endotoxin by limulus amebocyte lysate, Methods Mol Med, 36 (2000) 3-12.

[24] D.W. Bates, J. Parsonnet, P.A. Ketchum, E.B. Miller, T.J. Novitsky, K. Sands, P.L. Hibberd, P.S. Graman, P.N. Lanken, J.S. Schwartz, K. Kahn, D.R. Snydman, R. Moore, E. Black, R. Platt, Limulus amebocyte lysate assay for detection of endotoxin in patients with sepsis syndrome. AMCC Sepsis Project Working Group, Clin Infect Dis, 27 (1998) 582-591.

[25] M.D. Clayman, A. Raymond, D. Colen, C. Moffitt, C. Wolf, E.G. Neilson, The Limulus amebocyte lysate assay. A rapid and sensitive method for diagnosing early gram-negative peritonitis in patients undergoing continuous ambulatory peritoneal dialysis, Arch Intern Med, 147 (1987) 337-340.

[26] R. Vassallo, A.H. Limper, Fungal beta-glucan can yield false-positive results with the limulus amebocyte lysate endotoxin assay, Chest, 116 (1999) 583-584.

[27] H. Therisod, V. Labas, M. Caroff, Direct microextraction and analysis of rough-type lipopolysaccharides by combined thin-layer chromatography and MALDI mass spectrometry, Anal Chem, 73 (2001) 3804-3807.

[28] J. Lukasiewicz, W. Jachymek, T. Niedziela, L. Kenne, C. Lugowski, Structural analysis of the lipid A isolated from Hafnia alvei 32 and PCM 1192 lipopolysaccharides, Journal of Lipid Research, 51 (2010) 564-574.

[29] A. Kilar, A. Dornyei, B. Kocsis, Structural Characterization of Bacterial Lipopolysaccharides with Mass Spectrometry and on- and Off-Line Separation Techniques, Mass Spectrom Rev, 32 (2013) 90-117.

[30] J.P. Pais de Barros, T. Gautier, W. Sali, C. Adrie, H. Choubley, E. Charron, C. Lalande, N. Le Guern, V. Deckert, M. Monchi, J.P. Quenot, L. Lagrost, Quantitative lipopolysaccharide analysis using HPLC/MS/MS and its combination with the limulus amebocyte lysate assay, J Lipid Res, 56 (2015) 1363-1369.

[31] A. Sonesson, L. Larsson, A. Schutz, L. Hagmar, T. Hallberg, Comparison of the limulus amebocyte lysate test and gas chromatography-mass spectrometry for measuring lipopolysaccharides (endotoxins) in airborne dust from poultry-processing industries, Appl Environ Microbiol, 56 (1990) 1271-1278.

[32] A.P. de Santana, G.R. Noleto, P.A.J. Gorin, L.M. de Souza, M. Iacomini, G.L. Sassaki, GC-MS detection and quantification of lipopolysaccharides in polysaccharides through 3-O-acetyl fatty acid methyl esters, Carbohyd Polym, 87 (2012) 2730-2734.

[33] J.H. Gross, Direct analysis in real time-a critical review on DART-MS, Analytical and Bioanalytical Chemistry, 406 (2014) 63-80. 
[34] R.B. Cody, J.A. Laramee, J.M. Nilles, H.D. Durst, Direct analysis in real time (DART ${ }^{\mathrm{tm}}$ ) mass spectrometry JEOL news, 40 (2005) 8-12.

[35] W. Romao, L.V. Tose, B.G. Vaz, S.G. Sama, R. Lobinski, P. Giusti, H. Carrier, B. Bouyssiere, Petroleomics by Direct Analysis in Real Time-Mass Spectrometry, J Am Soc Mass Spectrom, 27 (2016) 182-185.

[36] N. Hayeck, S. Ravier, R. Gemayel, S. Gligorovski, I. Poulet, J. Maalouly, H. Wortham, Validation of Direct Analysis Real Time source/Time-of-Flight Mass Spectrometry for organophosphate quantitation on wafer surface, Talanta, 144 (2015) 1163-1170.

[37] R.L. Self, Direct analysis in real time-mass spectrometry (DART-MS) for rapid qualitative screening of toxic glycols in glycerin-containing products, J Pharm Biomed Anal, 80 (2013) 155-158.

[38] H.M. Park, H.J. Kim, Y.P. Jang, S.Y. Kim, Direct Analysis in Real Time Mass Spectrometry (DART-MS) Analysis of Skin Metabolome Changes in the Ultraviolet B-Induced Mice, Biomol Ther (Seoul), 21 (2013) 470-475.

[39] R.W. Jones, R.B. Cody, J.F. McClelland, Differentiating writing inks using direct analysis in real time mass spectrometry, J Forensic Sci, 51 (2006) 915-918.

[40] T. Higashide, K. Sugiyama, Use of viscoelastic substance in ophthalmic surgery - focus on sodium hyaluronate, Clin Ophthalmol, 2 (2008) 21-30.

[41] H.W. Flynn, I.U. Scott, T.G. Murray, W.J. Feuer, J.C. Schiffman, Intraoperative use of perfluoro-Noctane in the repair of complex retinal detachment: A prospective, multicenter, observational study, Invest Ophth Vis Sci, 42 (2001) S432-S432.

[42] M. Rofail, L.R. Lee, Perfluoro-N-octane as a postoperative vitreoretinal tamponade in the management of giant retinal tears, Retina-J Ret Vit Dis, 25 (2005) 897-901.

[43] L. Janco, K. Tkacova Villemova, M. Ondrejkova, R. Vida, M. Bartos, M. Mesarosova, [Retinal tamponade with silicone oil - long term results], Cesk Slov Oftalmol, 70 (2014) 178-182.

[44] N. Hong, B.S. Huang, J.P. Tong, Primary silicone oil tamponade and internal limiting membrane peeling for retinal detachment due to macular hole in highly myopic eyes with chorioretinal atrophy, BMC Ophthalmol, 15 (2015) 165.

[45] L.G. Song, A.B. Dykstra, H.F. Yao, J.E. Bartmess, Ionization Mechanism of Negative lon-Direct Analysis in Real Time: A Comparative Study with Negative lon-Atmospheric Pressure Photoionization, J Am Soc Mass Spectr, 20 (2009) 42-50.

[46] H. Yang, L. Shi, W. Yao, Y. Wang, L. Huang, D. Wan, S. Liu, Differentiation of Disaccharide Isomers by Temperature-Dependent In-Source Decay (TDISD) and DART-Q-TOF MS/MS, J Am Soc Mass Spectrom, 26 (2015) 1599-1605.

[47] Y. Wang, L. Liu, L. Ma, S. Liu, Identification of saccharides by using direct analysis in real time (DART) mass spectrometry, Int J Mass Spectrom, 357 (2014) 51-57.

[48] D.S. Saang'onyo, D.L. Smith, Optimization of direct analysis in real time (DART) linear ion trap parameters for the detection and quantitation of glucose, Rapid communications in mass spectrometry : RCM, 26 (2012) 385-391.

[49] E.S. Chernetsova, G.E. Morlock, Assessing the capabilities of direct analysis in real time mass spectrometry for 5-hydroxymethylfurfural quantitation in honey, Int J Mass Spectrom, 314 (2012) 22-32.

[50] E.S. Chernetsova, M.V. Ovcharov, G.V. Zatonskii, R.A. Abramovich, I.A. Revelskii, Clafication of the composition of $[\mathrm{M}+18](+)$ ions in DART mass spectra of polyethylene glycol using high-resolution mass spectrometry, J Anal Chem+, 66 (2011) 1348-1351.

[51] P. Nemes, W.J. Hoover, D.A. Keire, High-throughput differentiation of heparin from other glycosaminoglycans by pyrolysis mass spectrometry, Anal Chem, 85 (2013) 7405-7412.

[52] I.M. Lanzl, R.L. Merte, Silicone oil bubbles in ophthalmic viscosurgical devices, J Cataract Refract Surg, 28 (2002) 2069. 
[53] A. Ohrstrom, B. Svensson, S. Tegenfeldt, C. Celiker, B. Lignell, Silicone oil content in ophthalmic viscosurgical devices, J Cataract Refract Surg, 30 (2004) 1278-1280.

[54] E.P. Solov'eva, S.A. Muslimov, [Silicone oil tamponade as a risk factor of complications], Vestn Oftalmol, 129 (2013) 28-31.

[55] A. Lanzarotta, C.M. Kelley, Forensic Analysis of Human Autopsy Tissue for the Presence of Polydimethylsiloxane (Silicone) and Volatile Cyclic Siloxanes using Macro FT-IR, FT-IR Spectroscopic Imaging and Headspace GC-MS, J Forensic Sci, 61 (2016) 867-874.

[56] P.J. Tomlins, M.G. Woodcock, N. Spencer, G.R. Kirkby, Nuclear magnetic resonance analysis of emulsified silicone oil RMN-3 (Oxane HD), The British journal of ophthalmology, 91 (2007) 1379-1381.

[57] S.V. Kala, E.D. Lykissa, M.L. Russell, Detection and Characterization of Poly(dimethylsiloxane)s in Biological Tissues by GC/AED and GC/MS, Anal Chem, 69 (1997) 1269-1272.

[58] J.H. Gross, Polydimethylsiloxane-based wide-range mass calibration for direct analysis in real-time mass spectrometry, Anal Bioanal Chem, 405 (2013) 8663-8668.

[59] J.H. Gross, Polydimethylsiloxane extraction from silicone rubber into baked goods detected by direct analysis in real time mass spectrometry, Eur J Mass Spectrom (Chichester, Eng), 21 (2015) 313-319. [60] A. Jakob, E.A. Crawford, J.H. Gross, Detection of polydimethylsiloxanes transferred from siliconecoated parchment paper to baked goods using direct analysis in real time mass spectrometry, Journal of mass spectrometry : JMS, 51 (2016) 298-304. 
Table 1. Major fragments for endotoxin by DART-LTQ (ion trap) MS and proposed chemical formula for different ions with exact mass from an accurate mass Q-TOF MS.

\begin{tabular}{llllc}
\hline $\begin{array}{l}m / z \\
\text { value }\end{array}$ & $\begin{array}{l}\text { Chemical } \\
\text { Formula }\end{array}$ & $\begin{array}{l}\text { Proposed } \\
\text { Ion form }\end{array}$ & $\begin{array}{l}\text { Exact } \\
m / z \text { value }\end{array}$ & $\begin{array}{c}\text { Mass error } \\
(\mathrm{ppm})\end{array}$ \\
\hline $\mathbf{1 1 7}$ & $\mathrm{C}_{5} \mathrm{H}_{9} \mathrm{O}_{3}$ & {$\left[\mathrm{M}-\mathrm{H}_{2} \mathrm{O}-45\right]^{+}$} & 117.0565 & 11.0 \\
$\mathbf{1 2 7}$ & $\mathrm{C}_{6} \mathrm{H}_{7} \mathrm{O}_{3}$ & {$\left[\mathrm{M}-3 \mathrm{H}_{2} \mathrm{O}+\mathrm{H}\right]^{+}$} & 127.0407 & 9.4 \\
$\mathbf{1 4 5}$ & $\mathrm{C}_{6} \mathrm{H}_{9} \mathrm{O}_{4}$ & {$\left[\mathrm{M}-\mathrm{H}_{2} \mathrm{O}+\mathrm{H}^{+}\right.$} & 145.0514 & 8.6 \\
$\mathbf{1 6 3}$ & $\mathrm{C}_{6} \mathrm{H}_{11} \mathrm{O}_{5}$ & {$\left[\mathrm{M}-\mathrm{H}_{2} \mathrm{O}+\mathrm{H}\right]^{+}$} & 163.0619 & 7.6 \\
$\mathbf{1 8 0}$ & $\mathrm{C}_{6} \mathrm{H}_{14} \mathrm{NO}_{5}$ & {$\left[\mathrm{M}+\mathrm{NH}_{4}-\mathrm{H}_{2} \mathrm{O}\right]^{+}$} & 180.0882 & 5.7 \\
$\mathbf{1 9 8}$ & $\mathrm{C}_{6} \mathrm{H}_{16} \mathrm{NO}_{6}$ & {$\left[\mathrm{M}+\mathrm{NH}_{4}\right]^{+}$} & 198.0984 & 3.3 \\
$\mathbf{2 7 1}$ & $\mathrm{C}_{12} \mathrm{H}_{15} \mathrm{O}_{7}$ & {$\left[\mathrm{~N}-4 \mathrm{H}_{2} \mathrm{O}+\mathrm{H}\right]^{+}$} & 271.0840 & 8.1 \\
$\mathbf{2 8 9}$ & $\mathrm{C}_{12} \mathrm{H}_{17} \mathrm{O}_{8}$ & {$\left[\mathrm{~N}-3 \mathrm{H}_{2} \mathrm{O}+\mathrm{H}\right]^{+}$} & 289.0933 & 2.2 \\
$\mathbf{3 0 7}$ & $\mathrm{C}_{12} \mathrm{H}_{19} \mathrm{O}_{9}$ & {$\left[\mathrm{~N}-2 \mathrm{H}_{2} \mathrm{O}+\mathrm{H}\right]^{+}$} & 307.1033 & 1.4 \\
$\mathbf{3 2 5}$ & $\mathrm{C}_{12} \mathrm{H}_{21} \mathrm{O}_{10}$ & {$\left[\mathrm{~N}-\mathrm{H}_{2} \mathrm{O}+\mathrm{H}\right]^{+}$} & 325.1130 & -2.4 \\
$\mathbf{3 4 2}$ & $\mathrm{C}_{12} \mathrm{H}_{24} \mathrm{NO}_{10}$ & $\left.\left[\mathrm{~N}-\mathrm{H}_{2} \mathrm{O}+\mathrm{NH}\right]_{4}\right]^{+}$ & 342.1390 & -2.9 \\
$\mathbf{3 4 3}$ & $\mathrm{C}_{12} \mathrm{H}_{23} \mathrm{O}_{11}$ & {$\left[\mathrm{~N}+\mathrm{H}^{+}\right.$} & 343.1240 & 0.0 \\
$\mathbf{3 6 0}$ & $\mathrm{C}_{12} \mathrm{H}_{26} \mathrm{NO}_{11}$ & {$[\mathrm{~N}+\mathrm{NH}]^{+}$} & 360.1489 & -4.6 \\
$\mathbf{1 1 9}$ & $\mathrm{C}_{4} \mathrm{H}_{7} \mathrm{O}_{4}$ & {$[\mathrm{M}-60-\mathrm{H}]^{-}$} & 119.0345 & 0.7 \\
$\mathbf{1 4 3}$ & $\mathrm{C}_{6} \mathrm{H}_{7} \mathrm{O}_{4}$ & {$\left[\mathrm{M}-2 \mathrm{H}_{2} \mathrm{O}-\mathrm{H}\right]^{-}$} & 143.0344 & -0.5 \\
$\mathbf{1 6 1}$ & $\mathrm{C}_{6} \mathrm{H}_{9} \mathrm{O}_{5}$ & {$\left[\mathrm{M}-\mathrm{H}_{2} \mathrm{O}-\mathrm{H}\right]^{-}$} & 161.0451 & -.04 \\
$\mathbf{1 7 9}$ & $\mathrm{C}_{6} \mathrm{H}_{11} \mathrm{O}_{6}$ & {$[\mathrm{M}-\mathrm{H}]^{-}$} & 179.0548 & -4.5 \\
$\mathbf{2 2 1}$ & $\mathrm{C}_{8} \mathrm{H}_{13} \mathrm{O}_{7}$ & See SI & 221.0650 & -7.4 \\
$\mathbf{3 2 3}$ & $\mathrm{C}_{12} \mathrm{H}_{19} \mathrm{O}_{10}$ & {$\left[\mathrm{~N}-\mathrm{H}_{2} \mathrm{O}-\mathrm{H}\right]^{-}$} & 323.0970 & -2.8 \\
$\mathbf{3 4 1}$ & $\mathrm{C}_{11} \mathrm{H}_{21} \mathrm{O}_{11}$ & {$[\mathrm{~N}-\mathrm{H}]^{-}$} & 341.1069 & -4.6 \\
\hline
\end{tabular}

Note: $m / z$ values in the first column were obtained from a low selectivity ion trap MS, while exact $\mathrm{m} / z$ values (the fourth column) were acquired from a high resolution Q-TOF MS. The peaks listed in the Table can be used as gold standards for the detection of endotoxin in different matrices. $\mathrm{M}$ indicates a chemical formula of $\mathrm{C}_{6} \mathrm{H}_{12} \mathrm{O}_{6}$ for monosaccharide; $\mathrm{N}$ indicates a chemical formula of $\mathrm{C}_{12} \mathrm{H}_{22} \mathrm{O}_{11}$ for disaccharide; 45 indicates a loss of $\mathrm{COOH}, 60$ indicates a loss of $2 \mathrm{CHOH}$ from hexose. Please see supporting information (SI) Fig. S3 for the proposed structures of ions $\mathrm{m} / \mathrm{z} 117, \mathrm{~m} / \mathrm{z} 119$ and $\mathrm{m} / \mathrm{z} 221$. 


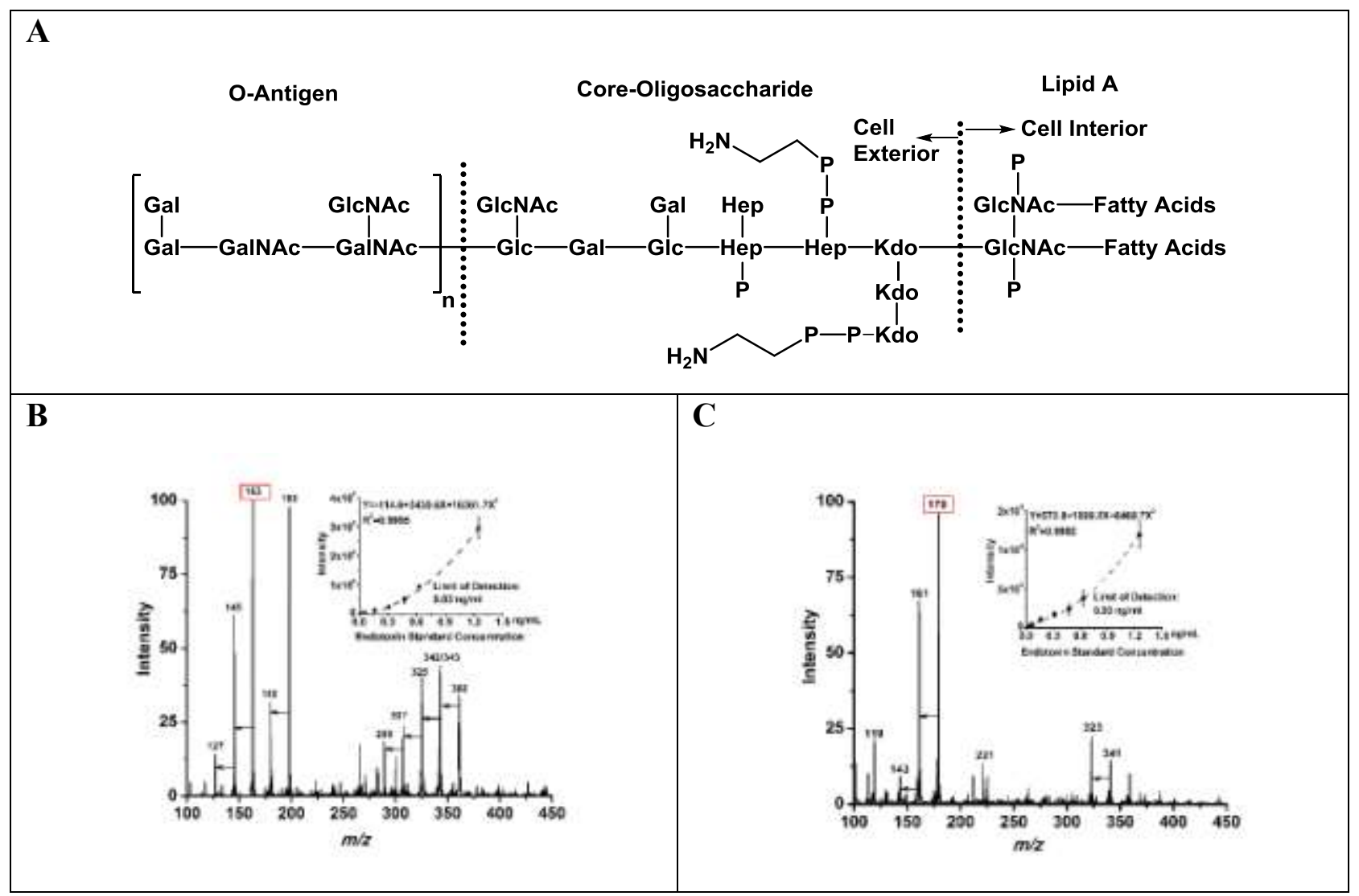

Fig. 1. The scheme of chemical structure of endotoxin is shown in 1A (Gal: galactose, Glc: glucose, GalNAc: $N$-acetyl-galactosamine, GlcNAc: $N$-acetyl-glucosamine, Hep: L-glycerol-Dmanno-heptose, KDO: 2-keto-3-deoxyoctonic acid). 1B and $\mathbf{1 C}$ show mass spectra of $0.62 \mathrm{ng}$ $\mathrm{mL}^{-1}$ endotoxin in positive mode and negative mode respectively, where major peaks on the spectra were annotated with $\mathrm{m} / \mathrm{z}$ values and the unlabeled arrows indicate consecutive water losses. Corresponding calibration curves are listed on the upper right hand corner of each spectrum. Lower limit of detection was established at $0.03 \mathrm{ng} \mathrm{mL} L^{-1}$ or equivalent to $0.3 \mathrm{EU} \mathrm{mL}^{-1}$ endotoxin using the intensity of $\mathrm{m} / \mathrm{z} 163$ in positive mode and intensity of $\mathrm{m} / \mathrm{z} 179$ in negative mode. Triplicate measurements were averaged for each data point on the curve. 
A

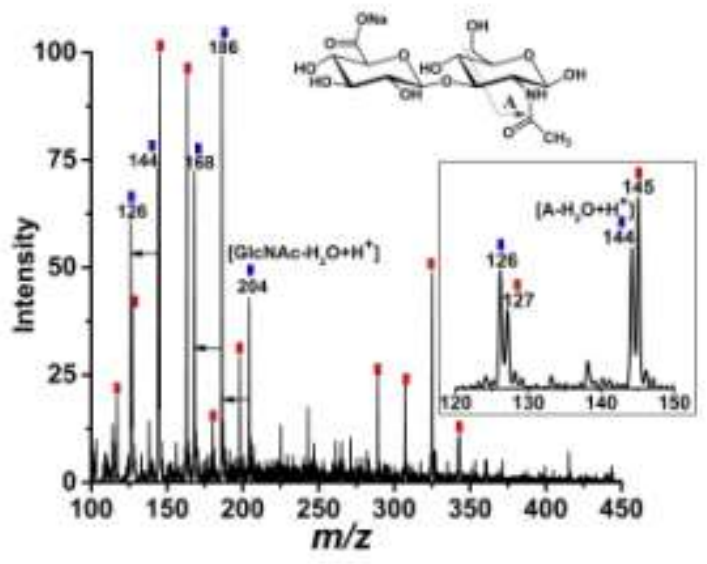

B

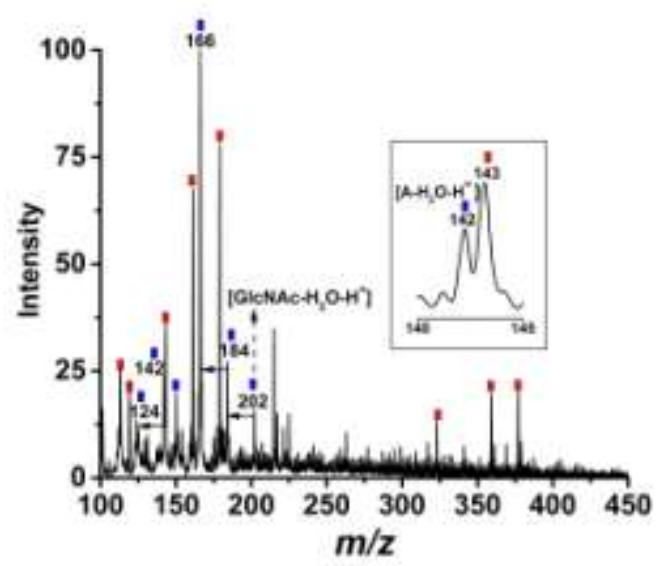

Fig. 2. Mass spectra for the mixture of endotoxin and sodium hyaluronate (NaHA) in positive (2A) and negative (2B) modes, respectively. The displayed sample contains $10 \mathrm{ng} \mathrm{mL} \mathrm{m}^{-1}$ endotoxin and 1 to 5 weight ratio of $\mathrm{NaHA}$ in 50:50 methanol: water mixture. Red square labeled $\mathrm{m} / \mathrm{z}$ ions indicate endotoxin signal, NaHA generated characteristic peaks in the same region are labeled as blue squares, where the proposed structures are annotated using its repeating disaccharide unit. Inserted windows show zoomed mass regions to display the detailed mass signal difference for endotoxin and NaHA. Unlabeled arrows indicate water loss for NaHA. 


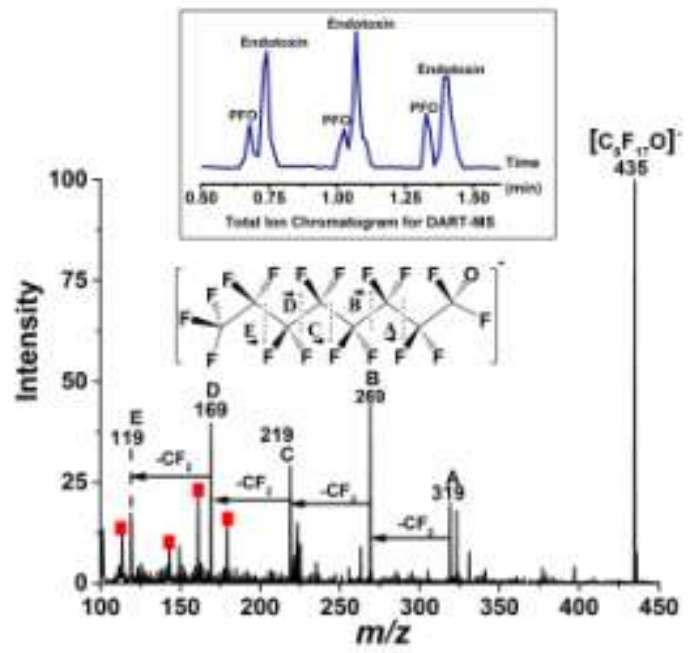

Fig. 3. Mass spectrum acquired for the mixture of endotoxin and perfluoro-n-octane (PFO) in negative ion mode. $1 \mu \mathrm{L}$ PFO was pipetted on top of $1 \mu \mathrm{L}$ of $1.25 \mathrm{ng} \mathrm{mL}^{-1}$ of aqueous endotoxin on a dip-it-tip, and the mixture was analyzed by DART-MS immediately through manual sampling. PFO fragmentation pattern was obtained by applying $30 \mathrm{~V}$ in-source fragmentation; red squares indicate the major endotoxin signals at $400^{\circ} \mathrm{C}$ DART analysis. During the DART ionization, one of the fluorine's of PFO is replaced by an oxygen atom to produce the most intense peak on the spectrum $\left(\mathrm{C}_{8} \mathrm{H}_{17} \mathrm{O}^{-}\right)$. 


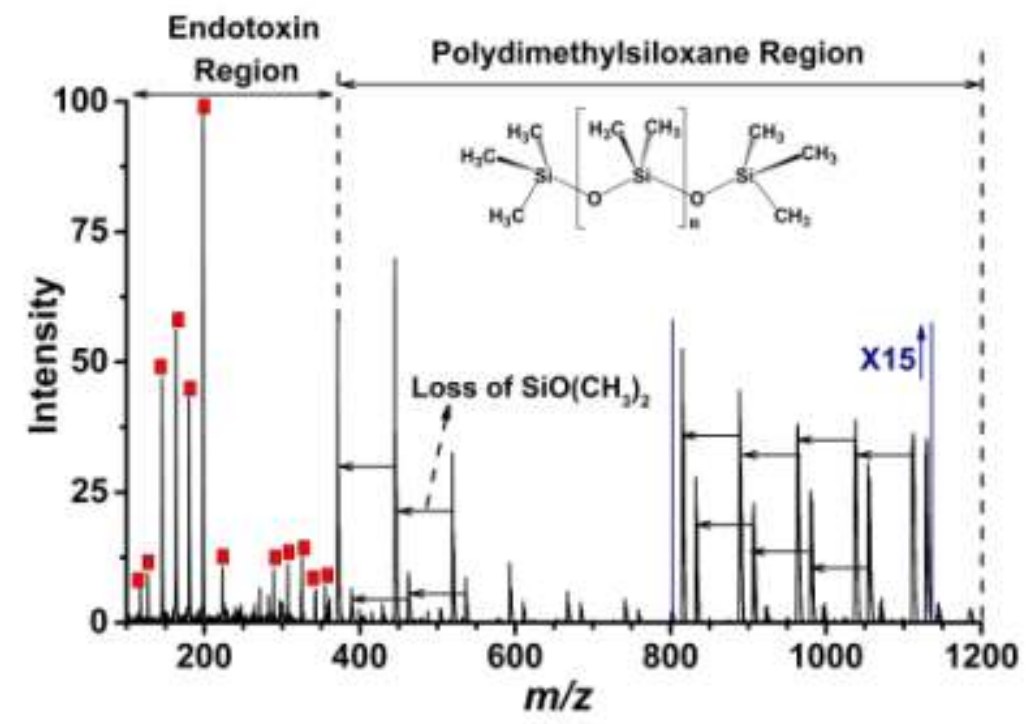

Fig. 4. Mass spectrum for a mixture of endotoxin and polydimethylsiloxane standard in positive ion mode. $1 \mu \mathrm{L}$ of $1.25 \mathrm{ng} \mathrm{mL}^{-1}$ of aqueous endotoxin and $1 \mu \mathrm{L}$ of 1 to 100 dilution of silicone oil in hexane were spotted on top of each other to the bottom of dip-it tip. Liquid sample was air dried before the DART-MS analysis at $400^{\circ} \mathrm{C}$. Two different mass distributions were observed for endotoxin (red square highlighted) and polydimethylsiloxane. $\mathrm{Y}$ axis intensity scale is zoomed for 15 fold between $\mathrm{m} / \mathrm{z} 800$ to $\mathrm{m} / \mathrm{z} 1150$; loss of $\mathrm{SiO}\left(\mathrm{CH}_{3}\right)_{2}$ was a common fragmentation observed for polydimethylsiloxane (indicated by horizontal arrows on the spectrum). 


\section{Graphical Abstract}

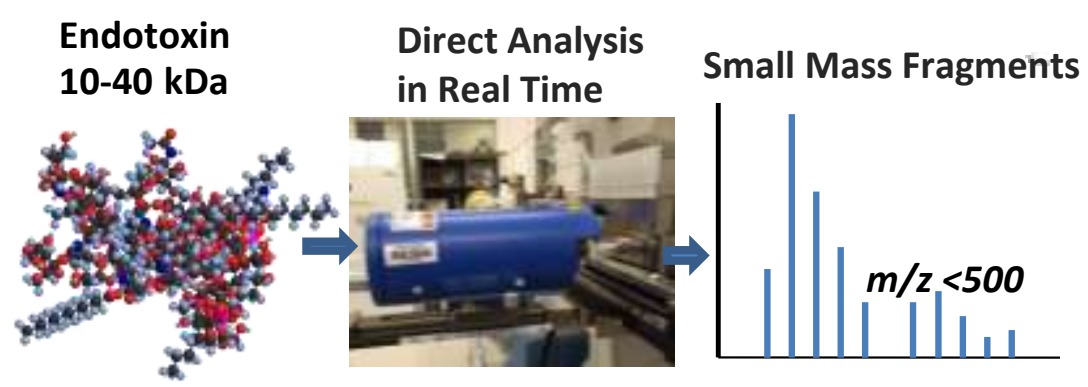

